\title{
Outcome of implantable loop recorder evaluation
}

\author{
Peter M. Magnusson ${ }^{1,2}$, Maciej Olszowka ${ }^{3}$, Marita Wallhagen ${ }^{4}$, Hirsh Koyi ${ }^{2,5}$ \\ ${ }^{1}$ Cardiology Research Unit, Department of Medicine, Karolinska Institutet, Stockholm, Sweden \\ ${ }^{2}$ Center for Research and Development, Uppsala University/Region Gävleborg, Gävle, Sweden \\ ${ }^{3}$ Department of Medical Sciences, Uppsala Clinical Research Centre, Uppsala University, Uppsala, Sweden \\ ${ }^{4}$ Department of Building, Energy and Environmental Engineering, University of Gävle, Gävle, Sweden \\ ${ }^{5}$ Department of Medicine, Karolinska Institutet, Stockholm, Sweden
}

\begin{abstract}
Background: The aim of this study was to evaluate implantable loop recorders (ILRs) in an unselected cohort in order to determine diagnostic yield, time to pacemaker/implantable cardioverter-defibrillator (ICD) implantation, predictors thereof, safety issues, and syncope management including usage of preceding diagnostic tools.

Methods: Patients who underwent ILR evaluation in any of three centers in Region Gävleborg, Sweden, between April 2007 and April 2013 were included and their medical records retrieved. Logistic regression was used to evaluate predictors of pacemaker/ICD outcome expressed as odds ratios (ORs) and Kaplan-Meier estimates for time-dependent analysis.

Results: A total of 173 patients (52.6\% females) with a mean age of 56.2 years received an ILR during a mean follow-up of 605 days. In the 146 patients evaluated for syncope/presyncope, $28.1 \%$ received a pacemaker $(n=39)$ or ICD $(n=2)$. The cumulative incidence at 6,12 , and 18 months were $8.8 \%$, $21.3 \%$, and $26.7 \%$, respectively. Age $>75$ years was the only significant predictor for outcome $(p=0.010)$ and the following variables showed a tendency toward significance: abnormal elevation of the biomarker $B$-type natriuretic peptide (OR 2.05, $p=0.100)$, a history of trauma (OR 1.71, $p=0.179)$, and pathologic electrocardiogram (OR 1.68, $p=0.231$ ). A computerized tomography of the skull was performed in $52.1 \%$ of the syncope cases.

Conclusions: In syncope evaluation in an unselected cohort, $28.1 \%$ were diagnosed with an arrhythmia necessitating a pacemaker/ICD. The only significant predictor was advanced age. Time to diagnosis is unpredictable and prolonged ILR monitoring is warranted in addition to optimal use of other diagnostic tools. (Cardiol J 2018; 25, 3: 363-370)
\end{abstract}

Key words: cardiac arrhythmia, implantable loop recorder, electrocardiography monitoring, pacemaker, syncope

\section{Introduction}

Cardiac arrhythmias may be challenging to detect and an implantable loop recorder (ILR) offers prolonged diagnostic evaluation. Syncope is a transient loss of consciousness caused by cerebral hypoperfusion and typically occurs suddenly, has a short duration and complete recovery [1]. In syn- cope caused by a cardiac arrhythmia, the prognosis is worse and up to a third of patients die within a year [2]; elderly patients seem to be especially at risk [3]. However, independent of its cause and prognosis, syncope may adversely affect quality of life because of anxiety, restricted activities like driving, and the risk of trauma. For patients with suspected syncope, it is crucial to get a detailed

Address for correspondence: Peter Magnusson, MD, Cardiology Research Unit, Department of Medicine, Karolinska Institutet, Karolinska University Hospital/Solna, SE-171 76 Stockholm, Sweden, tel: +46(0)705 089407,

fax: +46(0)26 154255, e-mail: peter.magnusson@regiongavleborg.se

Received: 14.04.2017 Accepted: 04.08.2017 
history and extensive evaluation is sometimes necessary, including the use of an ILR [4]. Almost half of the population will faint at least once over the course of a lifetime [5], with peak incidences in adolescence and old age, and syncope accounts for 1-3\% of emergency department visits [2]. Even though guidelines stress the role of ILR evaluation in syncope, in actual clinical practice the number of patients indicated for an ILR is about 4 times higher than the number who actually receive one and about one-third of those who do receive an ILR do not meet the criteria for one as set forth by the guidelines $[1,6]$. At a tertiary center, ILRs were used in $5 \%$ of referrals for syncope, in $28 \%$ of cases of unexplained syncope [7] and in 1\% of emergency visits [8]. In addition to syncope management, ILR are used to detect atrial fibrillation (AF) or explain symptoms such as palpitations, dizziness, and falls [1, 9-11]. Among syncope patients an ILR may reveal a significant bradycardia (asystole, sinus arrest, high-degree atrioventricular block) or a ventricular tachycardia possibly requiring pacemaker or implantable cardioverter-defibrillator (ICD) treatment, respectively [12, 13]. ILRs may also detect supraventricular tachycardias, which may result in such treatments as antiarrhythmic drug therapy, anticoagulation therapy, or an ablation procedure. The absence of cardiac arrhythmia may lead to further investigation or reassurance after a long-term follow-up period. Approximately a fifth of the patients in reported cohorts over the last few years underwent pacemaker implantation due to ILR findings of bradycardia [10, 14-17]. However these studies may be prone to selection bias as they were conducted at tertiary centers, which act as referral centers, while other studies use a prospective design, including specific protocols for disease management. Therefore, ILR evaluation from these studies may not necessarily be generalized to other settings.

The objectives of this ILR evaluation were to determine diagnostic yield, time to pacemaker/ICD and predictors thereof, safety issues, and syncope management, including the use of preceding diagnostic tools.

\section{Methods}

\section{Setting}

All consecutive patients who underwent an ILR procedure in Region Gävleborg between April 2007 and April 2013 were included. Region Gävleborg has a current catchment area of 281,815 inhabitants and 3 hospitals (Bollnäs, Gävle, and
Hudiksvall), which are all non-university hospitals that evaluate syncope patients, including the use of ILR procedures, as a diagnostic tool [18].

\section{Data sources and ethics}

The patients were identified using the medical record system, including the hospital-based surgery database that covers all ILR procedures. The daily updated Swedish population Census Bureau Register was used to ascertain if the patient was deceased and living patients were contacted (including three reminders and phone contact) to gain informed consent in order to read the relevant portions of their medical records. Data acquisition according to a predefined protocol of variables was performed by an experienced physician (MO) and validated by a cardiologist (PM). The Regional Ethical Committee in Uppsala approved the study (protocol number 2016/098), which was conducted in compliance with the Declaration of Helsinki.

\section{Statistical analysis}

Descriptive data were expressed as frequencies and percentages. Continuous variables were summarized as means including standard deviations (SDs), median, $25^{\text {th }}$, and $75^{\text {th }}$ percentiles, and compared using t-tests. Categorical variables were compared using $\chi^{2}$ test. Kaplan-Meier analyses and cumulative incidences were used to report time from first ILR procedure to outcome. An odds ratio (OR) with a $95 \%$ confidence interval (CI) was calculated for a potential predictor of outcome in univariable analyses followed by a multivariable model using logistic regression. A two-sided p-value of $<0.05$ was considered statistically significant. The database in Excel 2010 (Microsoft Corporation, Redmond, WA) was imported into SPSS version 22 (IBM, Armonk, NY), and graphical presentation was made using $R$ (R Core Team, 2014).

\section{ILR procedure and follow-up}

The ILR device was typically inserted subcutaneously in the left parasternal area in a short procedure (a few minutes in duration) under local anesthesia. The patients received a Medtronic Reveal $^{\mathrm{TM}}$ ILR (Minneapolis, MN): Reveal Dx ${ }^{\mathrm{TM}}$, Reveal XT ${ }^{\mathrm{TM}}$, and Reveal Plus ${ }^{\mathrm{TM}}$ or a St. Jude Medical Confirm ${ }^{\mathrm{TM}}$ ILR (St. Paul, MN) $(\mathrm{n}=6)$. The remote monitoring function was available and used in 46 (26.6\%) of the cases, preferably during the latter period of the study. Activation of rhythm-storage can be done by the patient or a bystander but may also be carried out automatically according to programmed criteria of arrhythmias. Adjustment of 
Table 1. Clinical characteristics of 173 implantable loop recorder patients with regard to syncope and miscellaneous indications.

\begin{tabular}{lccc}
\hline Variable & All patients (n = 173) & Syncope (n = 146) & Miscellaneous (n = 27) \\
\hline Age, mean (standard deviation [SD]) & $56.2(\mathrm{SD} 19.9)$ & $56.4(\mathrm{SD} 20.8)$ & $54.8(\mathrm{SD} 14.1)$ \\
Male sex & $82(47.4 \%)$ & $67(45.9 \%)$ & $15(55.6 \%)$ \\
Diabetes & $8(4.6 \%)$ & $7(4.8 \%)$ & $1(3.7 \%)$ \\
Smoker & $24(13.9 \%)$ & $18(12.3 \%)$ & $6(22.2 \%)$ \\
Depression & $12(6.9 \%)$ & $12(8.2 \%)$ & $0(0 \%)$ \\
Polyneuropathy & $2(1.2 \%)$ & $2(1.4 \%)$ & $0(0 \%)$ \\
Migraine & $8(4.6 \%)$ & $7(4.8 \%)$ & $1(3.7 \%)$ \\
Epilepsy & $1(0.6 \%)$ & $1(0.7 \%)$ & $0(0 \%)$ \\
Stroke/transitory ischemic attack & $21(12.1 \%)$ & $15(10.3 \%)$ & $6(22.2 \%)$ \\
Antiarrhythmics & $58(33.5 \%)$ & $46(31.5 \%)$ & $12(44.4 \%)$ \\
Hypertension & $62(35.8 \%)$ & $53(36.3 \%)$ & $9(33.3 \%)$ \\
Reduced ejection fraction $(\leq 40 \%)$ & $3(1.7 \%)$ & $3(2.1 \%)$ & $0(0 \%)$ \\
Pathologic electrocardiogram & $51(29.5 \%)$ & $45(30.8 \%)$ & $6(22.2 \%)$ \\
Valvular heart disease & $11(6.4 \%)$ & $10(6.8 \%)$ & $1(3.7 \%)$ \\
Abnormal B-type natriuretic peptide & $47(27.2 \%)$ & $41(28.1 \%)$ & $6(22.2 \%)$ \\
Ventricular hypertrophy $\geq 15 \mathrm{~mm}$ & $2(1.2 \%)$ & $2(1.4 \%)$ & $0(0 \%)$ \\
Ischemic heart disease & $22(12.7 \%)$ & $20(13.7 \%)$ & $2(7.4 \%)$ \\
\hline
\end{tabular}

the programmed sensitivity setting was sometimes necessary. Atrial tachycardias were subdivided into $\mathrm{AF}$, atrial flutter, and ectopic atrial tachycardia.

\section{Results}

\section{Patient characteristics}

During the 6 -year period, a total of 210 patients received ILR evaluation and after consenting to participate in the study, $82.4 \%$ of these were analyzed $(n=173)$, whose baseline characteristics are summarized in Table 1 . Their mean age at ILR implant was 56.2 years and ranged from 16 to 90 years with a median of 60.0 years and $25^{\text {th }}$ percentiles 42 years, and $75^{\text {th }}$ percentiles 73 years. Around half $(\mathrm{n}=91 ; 52.6 \%)$ were females and mean age did not differ with regard to sex (58.6 years vs. 54.0 years, $\mathrm{p}=0.132$ ). Mean age and sex distribution were similar between the 3 centers (Gävle: $\mathrm{n}=90 ; 52.5 \% ; \mathrm{p}=0.433$; Hudiksvall: $\mathrm{n}=40$; $66.2 \% ; \mathrm{p}=0.450$ Bollnäs: $\mathrm{n}=43 ; 54.6 \%, \mathrm{p}=0.581$ ). The main indication for ILR evaluation was a composite indication of syncope $(\mathrm{n}=146 ; 84.4 \%)$, which included presyncope $(\mathrm{n}=11 ; 6.4 \%)$, single syncope $(n=24 ; 13.9 \%)$, or multiple syncope $(n=111$; $64.2 \%)$. Other indications of ILR were palpitations $(\mathrm{n}=15 ; 8.7 \%), \mathrm{AF} /$ cerebral infarction $(\mathrm{n}=6$; $3.5 \%)$, atrioventricular block $(\mathrm{n}=2 ; 1.2 \%)$, sinus node dysfunction $(\mathrm{n}=3 ; 1.7 \%)$, and nonsustained ventricular tachycardia $(\mathrm{n}=1 ; 0.6 \%)$.

\section{Diagnostic work-up of syncope}

In taking the history of patients with syncopal episodes, the following circumstances were noted: trauma $(n=51)$, prodromal symptoms $(n=67)$, seizure $(\mathrm{n}=10)$, and body posture (upright, $\mathrm{n}=70$; supine, $n=6$; sitting $n=69$; unknown, $n=28$ ). Several patients performed a computerized tomography $(\mathrm{CT})(\mathrm{n}=79)$ of the skull or cardiac magnetic resonance imaging $(\mathrm{n}=12)$ as part of syncope evaluation. A head-up tilt test (HUT) test was performed in 4 patients as part of the syncope evaluation. Notably, carotid sinus massage was carried out as part of clinical physical examination in 27 (15.6\%) patients at any time after a syncopal spell. Arrhythmia monitoring included Holter monitoring or ambulatory electrocardiogram (ECG) in the ward and in a minority of cases additional recorders were used, such as Zenicor ${ }^{\mathrm{TM}}(\mathrm{n}=14)$ or King of Hearts Express $^{\mathrm{TM}}(\mathrm{n}=5)$. In Table 2, diagnostic tools used before ILR is summarized with regard to syncope and miscellaneous indications.

\section{Follow-up time}

The follow-up time with ILR monitoring varied from 3 to 1,482 days. In total, the cohort was moni- 
Table 2. Diagnostics tools used before implantable loop recorder evaluation with regard to syncope and miscellaneous indications.

\begin{tabular}{lccc}
\hline Variables & All patients $(\mathbf{n}=173)$ & Syncope $(\mathbf{n}=146)$ & Miscellaneous (n= 27) \\
\hline Holter & $108(62.4 \%)$ & $87(59.6 \%)$ & $21(77.8 \%)$ \\
Exercise test & $98(56.6 \%)$ & $81(55.5 \%)$ & $17(63.0 \%)$ \\
Active standing test & $89(51.4 \%)$ & $87(59.6 \%)$ & $2(7.4 \%)$ \\
Head computer tomography & $79(45.7 \%)$ & $76(52.1 \%)$ & $3(11.1 \%)$ \\
Electroencephalogram & $58(33.5 \%)$ & $58(39.7 \%)$ & $0(0 \%)$ \\
Carotid sinus massage & $27(15.6 \%)$ & $27(18.5 \%)$ & $0(0 \%)$ \\
ZenicorTM (external loop recorder) & $14(8.1 \%)$ & $5(3.4 \%)$ & $9(33.3 \%)$ \\
Electrophysiological study & $13(7.5 \%)$ & $8(5.5 \%)$ & $5(18.5 \%)$ \\
External loop recorder & $5(2.9 \%)$ & $4(2.7 \%)$ & $1(3.7 \%)$ \\
Head-up tilt test & $4(2.3 \%)$ & $4(2.7 \%)$ & $0(0 \%)$ \\
\hline
\end{tabular}

tored during a total of 104,685 days (286.6 years). The mean follow-up was $605 \pm 409$ days. The decision to explant was based on the physician's judgment who decided an evaluation sufficient to reach a definite endpoint, such as device implant (pacemaker/ICD), initiation of pharmacological regimen, dosage changes to an existing drug regimen, electrophysiology study and ablation, patient death, or reassurance. It is noteworthy that several patients were monitored by ILR for more than 3 years, which is the estimated device longevity as reported by the manufacturer.

\section{Outcome}

The outcome of the ILR evaluation is depicted in Figure 1 as cumulative incidence with regard to time from implant. A total of 39 patients with syncope experienced a clinically relevant arrhythmia (sinus arrest/bradycardia, atrioventricular block, asystolia) and were recommended implantation of a permanent pacemaker (DDDR, VVIR, and AAIR devices in 29,9 , and 1 patients, respectively). In addition, 2 patients received an ICD due to ventricular tachycardia. This amounts to $28.1 \%$ who received a pacemaker or ICD during the total follow-up.

The cumulative incidence of pacemaker/ICD implant at 6,12 , and 18 months were $8.8 \%, 21.3 \%$, and $26.7 \%$, respectively. This time-to-event analysis is graphically presented in Figure 1.

\section{Predictors}

The only predictor of pacemaker/ICD outcome which correlated significantly with outcome was age $>75$ years. No other predictors of pacemaker/ ICD outcome (male sex, age $>75$ years, history of trauma at a syncope episode, ischemic heart disease, hypertension, antiarrhythmic agents

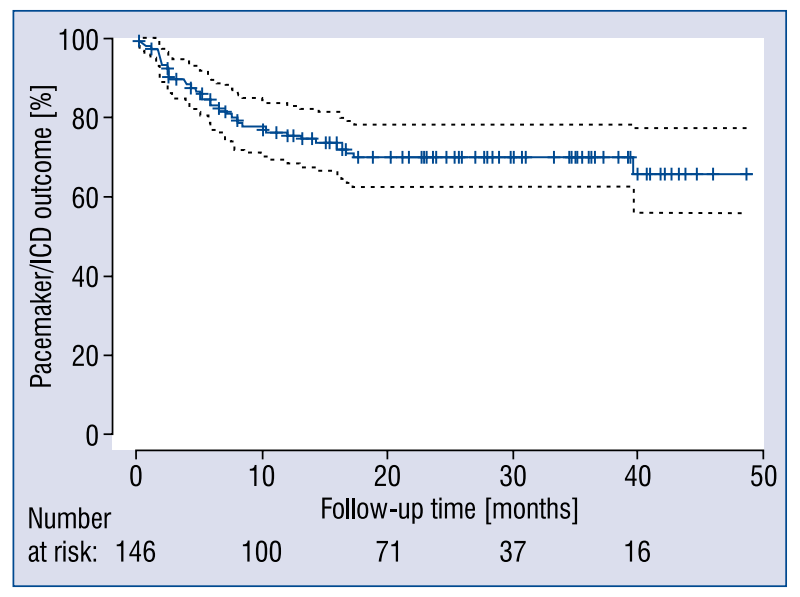

Figure 1. Kaplan-Meier curve of pacemaker/implantable cardioverter-defibrillator (ICD) outcome after implantable loop recorder evaluation.

(including beta-blockers), prodromal symptoms, stroke, ECG pathology, or abnormal heart failure marker (B-type natriuretic peptide/ $\mathrm{N}$-terminal pro-BNP [BNP/NT-proBNP]) correlated significantly $(\mathrm{p}<0.05)$ with outcome. The relative risk for pacemaker/ICD implant in patients aged $>75$ years was $44 \%$. A tendency for increased relative risk ( $p<0.20$ ) was observed for patients with a history of trauma (17\%) and abnormal BNP/NT-proBNP levels (25\%), while sex, ischemic heart disease, antiarrhythmic agents, prodromal symptoms, stroke, and pathologic ECG did not. None of these dichotomized categorical variables were significantly associated with outcome in univariable or multivariable analysis. The association between predictors and pacemaker/ICD outcome using a logistic regression model is summarized in Table 3. 
Table 3. Predictors of pacemaker or implantable defibrillator outcome among 146 patients evaluated with an implantable loop recorder due to syncope/presyncope.

\begin{tabular}{|c|c|c|c|c|c|c|c|}
\hline \multirow[t]{2}{*}{ Variable } & \multirow[b]{2}{*}{$\mathbf{N}(\%)$} & \multicolumn{3}{|c|}{ Univariable analysis } & \multicolumn{3}{|c|}{ Multivariable analysis } \\
\hline & & OR & $95 \% \mathrm{Cl}$ & $\mathbf{P}$ & OR & $95 \% \mathrm{Cl}$ & $\mathbf{P}$ \\
\hline Age $>75$ years & $37(25 \%)$ & 3.01 & $1.37-6.63$ & 0.010 & 2.61 & $1.10-6.24$ & 0.031 \\
\hline Male sex & $67(46 \%)$ & 1.03 & $0.50-2.12$ & 1.000 & & & \\
\hline Trauma & $51(35 \%)$ & 1.71 & $0.81-3.59$ & 0.179 & 0.59 & $0.28-1.27$ & 0.592 \\
\hline Stroke & $14(10 \%)$ & 2.08 & $0.67-6.41$ & 0.218 & & & \\
\hline Ischemic heart disease & $20(14 \%)$ & 1.11 & $0.40-3.13$ & 0.795 & & & \\
\hline Hypertension & $53(36 \%)$ & 1.02 & $0.48-2.15$ & 1.000 & & & \\
\hline Antiarrhythmics & $46(32 \%)$ & 1.19 & $0.55-2.55$ & 0.695 & & & \\
\hline Prodromal symptom & $67(46 \%)$ & 1.03 & $0.50-2.12$ & 1.000 & & & \\
\hline Pathologic electrocardiogram & $45(31 \%)$ & 1.68 & $0.79-3.58$ & 0.231 & & & \\
\hline Abnormal BNP & $41(28 \%)$ & 2.05 & $0.95-4.43$ & 0.100 & 0.72 & $0.30-1.71$ & 0.450 \\
\hline
\end{tabular}

BNP — B-type natriuretic peptide; $\mathrm{Cl}$ — confidence interval; OR — odds ratio

In a multivariable analysis using predictors from the univariable analysis with $\mathrm{p} \leq 0.100$ results, only advanced age $>75$ years emerges as a significant predictor; the same holds true if variables with significance level of $\mathrm{p} \leq 0.200$ are included.

Arrhythmias or other clinical scenarios were detected in a substantial portion of patients who had a miscellaneous indication for an ILR. In patients with a non-syncope indication for ILR, 2 patients received a pacemaker.

None of the analyzed patients had acomplication (i.e. infection) leading to premature explantation.

\section{Deaths during ILR monitoring}

In total, 6 patients died while being monitored by an ILR. One elderly patient with asymptomatic second-degree atrioventricular block Mobitz type II during gastroenteritis who died in the Emergency Department of cardiac arrest. One elderly patient with several comorbidities living in a nursing home had a history of suspected post-apoplectic epilepsy and died suddenly. The ILR of another elderly patient with polyneuropathy revealed $4 \mathrm{~s}$ of asystole at death. Two other patients with severe comorbidities died from metastatic cancer. A young patient with a phenotype of type 1 long QT syndrome and QT duration greater than $500 \mathrm{~ms}$ despite $200 \mathrm{mg}$ daily dosage of metoprolol therapy died suddenly and postmortem analysis confirmed the expected serum concentration of metoprolol; the cause of death was attributed to polymorphic ventricular arrhythmia.

\section{Discussion}

Syncope was the indication that led to the vast majority of ILR evaluations in this study and about a third $(28.1 \%)$ of patients with a history of unexplained presyncope or syncope went on to eventually receive a pacemaker or ICD after a thorough diagnostic work-up. The present findings fall in the higher end of results obtained from prior studies, where a $19 \%$ to $27 \%$ bradyarrhythmic etiology of syncope was observed [7, 8, 15, 19, 20]. It is important to note that the time span from ILR implantation until the point at which a clinically relevant arrhythmia is recorded can vary markedly among patients. This underscores the highly unpredictable course of cardiac syncope. A prior study reported that more than 1.5 years was needed for the ILR to capture clinically relevant information in about a quarter of syncopal or presyncopal patients [20]. Therefore, monitoring strategies should take a long-term view and devices should be able to monitor patients for a prolonged period of time.

In the largest prospective study to date of the use of ILRs in patients with unexplained syncope, $15.1 \%$ of patients received pacemakers, $6 \%$ ICDs, and $78 \%$ had overall guidance to diagnosis [11]. In the Spanish Reveal Registry, $31 \%$ of patients were treated according to ILR findings, of whom $21.3 \%$ received a pacemaker and $1.6 \%$ an ICD [17]. A randomized study of unexplained syncope patients found that $15.4 \%$ of patients (6/39) in the ILR group ended up being implanted with a pacemaker [21]. In an observational study at a tertiary care 
center in France, $21.1 \%$ of patients (20/95) with recurrent syncope received permanent pacemakers [15]. Two Italian studies from tertiary centers yielded pacemaker implant in $19.0 \%(11 / 58)$ [22] and $19.6 \%(11 / 56)$ [16], respectively. A recent British study from a university hospital reported $16.5 \%$ of patients $(\mathrm{n}=200)$ received pacemaker implants following ILR evaluation [14]. The present cohort had a higher proportion of permanent device implantation, even though the mean age in this study was lower than in the above-mentioned studies. However, the present study had a substantial subset of patients older than 75 years and this subgroup also had a significantly higher odds ratio for this outcome (device implantation). The sex distribution of our study was similar to these other studies. The lower proportion of pacemaker/ICD implantations that occurred in prospective studies may be explained by individual study requirements to adhere to a specific protocol, whereas in our study and in the Spanish Registry, clinicians were not obligated to follow any specific protocol [17]. This may mean that this study had higher specificity, on the one hand, but lower sensitivity on the other, in that guidelines related to device implant [6] are not rigorously translated into actual clinical practice [23].

Advanced age has been identified as a predictor of pacemaker/ICD outcome [22, 24, 25], which may be associated with an abnormal ECG and heart failure/structural heart disease [7, 26]. When syncope is caused by a sudden-onset arrhythmia resulting in cerebral hypoperfusion and abrupt loss of postural tone, trauma is more likely than with syncope caused by other mechanisms. In several studies, a history of trauma has been associated with cardiac syncope [14, 27, 28]. This study tended toward those findings, too. However, in a multivariable analysis only age $>75$ years fulfilled statistical significance of $p<0.05$, which highlights the difficulties in using predictors for clinical decision-making.

The Spanish Registry and the prospective PICTURE study show that diagnostic episodes often occur a year or more after ILR implant [11, 17]. The unpredictability of cardiac arrhythmias is vividly illustrated by the time-to-event analysis depicted in Figure 1 and support findings from another recent study analyzing pacemaker outcomes [16]. This clinically important finding supports the value of longer monitoring periods and advocates for the use of ILRs that can provide three or even more years of information. The extra resources required for extended follow-up may be met in part by nurse specialists and remote monitoring capabilities, making prolonged ILR evaluations cost effective [29]. The role of external loop recorders, which extend the conventional $24 \mathrm{~h}$ to $48 \mathrm{~h}$ capacity of Holter monitors, may be expanding as the syncopal population is a heterogeneous one; for example, external loop recorders may be appropriate first-line devices for patients experiencing palpitations or with suspected AF. Recent advances in software technology and photopletysmographic technology for monitoring AF may open the door to diagnostic tools such as smart-watches in disease management of certain patients [30].

The clinical management of patients with syncope, palpitations, AF, and dizziness remains challenging. These conditions require a multidisciplinary approach and increased knowledge among healthcare providers along the whole continuum of care for these patients. A knowledge and appreciation of each diagnostic tool and its individual role in syncopal evaluation is crucial in order to achieve optimal outcomes [31]. In this study, only $5.5 \%$ of patients underwent an electrophysiologic study, which reflects the unselected nature of this patient cohort. The present study was retrospective and thus there was no prospective protocol or algorithm for physicians to follow in guiding their work-flow. The same is probably true for HUT $(2.7 \%)$ and its diminishing role as reflected in the guidelines. In contrast, syncope was initially evaluated in this study by exercise testing (55.5\% of patients) and/ /or CT of the skull (52.1\%), which aligns with findings from the PICTURE trial [11]. These tests or scans were not actually indicated in many cases. For example, CT of the skull was often performed without signs of focal neurological signs, suspicion of epilepsy, or to exclude bleeding secondary to trauma. This use of CT scans might expose patients to unnecessary radiation and also represents an overuse of resources. Finally, it must be stressed that ILR solely offers diagnostics, not treatment, and risk stratification should be based on current guidelines, which sometimes recommend pacemaker or ICD implantation without the preceding finding of an arrhythmia [12, 13]. In fact, according to the guidelines for patient risk stratification, the patient with a long QT syndrome should have been considered for an ICD [13].

\section{Strengths and weaknesses}

The data from this triple-center observational study of ILR without referral center bias were retrospectively collected and validated. The advantage of this pragmatic approach is that clinical decision- 
making was not influenced by any management algorithms for inclusion and follow-up. In Sweden, all citizens are covered by national health insurance which includes ILRs making any socioeconomic impact on disease management less likely. Still, the ILR device may be considered costly, making some degree of patient selection necessary; it is unclear how this may have influenced the present study. Although this is a rather large ILR study, the sample size limits the study in terms of any interpretation of how predictors might be associated with outcomes.

\section{Conclusions}

In syncope evaluation in an unselected cohort of ILR patients, $28.1 \%$ required a pacemaker or an ICD due to the diagnosis of an arrhythmia. Advanced age was the only significant predictor for arrhythmia requiring device therapy. Time to diagnosis is unpredictable and prolonged ILR monitoring is warranted in addition to the optimal use of other diagnostic tools.

\section{Acknowledgements}

The authors acknowledge editing by Jo Ann LeQuang of LeQ Medical who reviewed the manuscript for American English use.

Funding: Region Gävleborg funded this research project.

\section{Conflict of interest: None declared}

\section{References}

1. Moya A, Sutton R, Ammirati F, et al. Task Force for the Diagnosis and Management of Syncope, European Society of Cardiology (ESC), European Heart Rhythm Association (EHRA), Heart Failure Association (HFA), Heart Rhythm Society (HRS). Guidelines for the diagnosis and management of syncope (version 2009). Eur Heart J. 2009; 30(21): 2631-2671, doi: 10.1093/eurheartj/ ehp298, indexed in Pubmed: 19713422.

2. Soteriades ES, Evans JC, Larson MG, et al. Incidence and prognosis of syncope. N Engl J Med. 2002; 347(12): 878-885, doi: 10.1056/NEJMoa012407, indexed in Pubmed: 12239256.

3. Colivicchi F, Ammirati F, Melina D. OESIL (Osservatorio Epidemiologico sulla Sincope nel Lazio) Study Investigators. Development and prospective validation of a risk stratification system for patients with syncope in the emergency department: the OESIL risk score. Eur Heart J. 2003; 9: 811-819.

4. Costantino G, Sun B, Barbic F, et al. Syncope clinical management in the emergency department: a consensus from the first international workshop on syncope risk stratification in the emergency department. Eur Heart J. 2016; 37(19): 1493-1498, doi: 10.1093/eurheartj/ehv378.
5. Ganzeboom KS, Mairuhu G, Reitsma JB, et al. Lifetime cumulative incidence of syncope in the general population: a study of 549 Dutch subjects aged 35-60 years. J Cardiovasc Electrophysiol. 2006; 17(11): 1172-1176, doi: 10.1111/j.1540-8167.2006.00595.x, indexed in Pubmed: 17074006.

6. Vitale E, Ungar A, Maggi R, et al. Discrepancy between clinical practice and standardized indications for an implantable loop recorder in patients with unexplained syncope. Europace. 2010; 12(10): 1475-1479, doi: 10.1093/europace/euq302, indexed in Pubmed: 20876604.

7. Solano A, Menozzi C, Maggi R, et al. Incidence, diagnostic yield and safety of the implantable loop-recorder to detect the mechanism of syncope in patients with and without structural heart disease. Eur Heart J. 2004; 25(13): 1116-1119, doi: 10.1016/j. ehj.2004.05.013, indexed in Pubmed:15231369.

8. Brignole M, Menozzi C, Bartoletti A, et al. A new management of syncope: prospective systematic guideline-based evaluation of patients referred urgently to general hospitals. Eur Heart J. 2006; 27(1): 76-82, doi: 10.1093/eurheartj/ehi647, indexed in Pubmed: 16272210.

9. Sanna T, Diener HC, Passman RS, et al. CRYSTAL AF Investigators. Cryptogenic stroke and underlying atrial fibrillation. $\mathrm{N} \mathrm{Engl}$ J Med. 2014; 370(26): 2478-2486, doi: 10.1056/NEJMoa1313600, indexed in Pubmed: 24963567.

10. Bhangu J, McMahon CG, Hall P, et al. Long-term cardiac monitoring in older adults with unexplained falls and syncope. Heart. 2016; 102(9): 681-686, doi: 10.1136/heartjnl-2015-308706, indexed in Pubmed: 26822427.

11. Edvardsson N, Frykman V, van Mechelen R, et al. PICTURE Study Investigators. Use of an implantable loop recorder to increase the diagnostic yield in unexplained syncope: results from the PICTURE registry. Europace. 2011; 13(2): 262-269, doi: 10.1093/europace/euq418, indexed in Pubmed:21097478.

12. Brignole M, Auricchio A, Baron-Esquivias G, et al. 2013 ESC Guidelines on cardiac pacing and cardiac resynchronization therapy: the Task Force on cardiac pacing and resynchronization therapy of the European Society of Cardiology (ESC). Developed in collaboration with the European Heart Rhythm Association (EHRA). Eur Heart J. 2013; 34(29): 2281-2329, doi: 10.1093/ eurheartj/eht150, indexed in Pubmed: 23801822.

13. Priori SG, Blomström-Lundqvist C, Mazzanti A, et al. 2015 ESC Guidelines for the management of patients with ventricular arrhythmias and the prevention of sudden cardiac death: The Task Force for the Management of Patients with Ventricular Arrhythmias and the Prevention of Sudden Cardiac Death of the European Society of Cardiology (ESC). Endorsed by: Association for European Paediatric and Congenital Cardiology (AEPC). Eur Heart J. 2015; 36: 2793-2867.

14. Ahmed N, Frontera A, Carpenter A, et al. Clinical Predictors of Pacemaker Implantation in Patients with Syncope Receiving Implantable Loop Recorder with or without ECG Conduction Abnormalities. Pacing Clin Electrophysiol. 2015; 38(8): 934-941, doi: 10.1111/pace.12666, indexed in Pubmed:25973599.

15. Pierre B, Fauchier L, Breard G, et al. Implantable loop recorder for recurrent syncope: influence of cardiac conduction abnormalities showing up on resting electrocardiogram and of underlying cardiac disease on follow-up developments. Europace. 2008; 10(4): 477-481, doi:10.1093/europace/eun039, indexed in Pubmed: 18325892.

16. Palmisano P, Accogli M, Zaccaria M, et al. Predictive factors for pacemaker implantation in patients receiving an implant- 
able loop recorder for syncope remained unexplained after an extensive cardiac and neurological workup. Int J Cardiol. 2013; 168(4): 3450-3457, doi:10.1016/j.ijcard.2013.04.179, indexed in Pubmed: 23701932.

17. Lacunza-Ruiz FJ, Moya-Mitjans A, Martínez-Alday J, et al. Implantable loop recorder allows an etiologic diagnosis in one-third of patients. Results of the Spanish reveal registry. Circ J. 2013; 77(10): 2535-2541, indexed in Pubmed: 23877732.

18. Statistics Sweden Database. Official population statistics. Kommunfakta Befolkningspyramid. 2016. www.scb.se.

19. Lombardi F, Calosso E, Mascioli G, et al. Utility of implantable loop recorder (Reveal Plus) in the diagnosis of unexplained syncope. Europace. 2005; 7(1): 19-24, doi: 10.1016/j. eupc.2004.09.003, indexed in Pubmed: 15670962.

20. Furukawa T, Maggi R, Bertolone C, et al. Additional diagnostic value of very prolonged observation by implantable loop recorder in patients with unexplained syncope. J Cardiovasc Electrophysiol. 2012; 23(1): 67-71, doi: 10.1111/j.1540-8167.2011.02133.x, indexed in Pubmed: 21777327.

21. Podoleanu C, DaCosta A, Defaye P, et al. FRESH investigators. Early use of an implantable loop recorder in syncope evaluation: a randomized study in the context of the French healthcare system (FRESH study). Arch Cardiovasc Dis. 2014; 107(10): 546-552, doi: 10.1016/j.acvd.2014.05.009, indexed in Pubmed: 25241220.

22. Maggi R, Rafanelli M, Ceccofiglio A, et al. Additional diagnostic value of implantable loop recorder in patients with initial diagnosis of real or apparent transient loss of consciousness of uncertain origin. Europace. 2014; 16(8): 1226-1230, doi: 10.1093/ europace/euu051, indexed in Pubmed: 24668513.

23. Kulakowski P, Lelonek M, Krynski T, et al. Prospective evaluation of diagnostic work-up in syncope patients: results of the PL-US registry. Europace. 2010; 12(2): 230-239, doi: 10.1093/ europace/eup367, indexed in Pubmed: 19919967.
24. Brignole M, Menozzi C, Maggi R, et al. The usage and diagnostic yield of the implantable loop-recorder in detection of the mechanism of syncope and in guiding effective antiarrhythmic therapy in older people. Europace. 2005; 7(3): 273-279, doi: 10.1016/j. eupc.2005.02.116, indexed in Pubmed:15878567.

25. Sarasin FP, Hanusa BH, Perneger T, et al. A risk score to predict arrhythmias in patients with unexplained syncope. Acad Emerg Med. 2003; 10(12): 1312-1317, indexed in Pubmed: 14644781.

26. Del Rosso A, Ungar A, Maggi R, et al. Clinical predictors of cardiac syncope at initial evaluation in patients referred urgently to a general hospital: the EGSYS score. Heart. 2008; 94(12): 1620-1626, doi: 10.1136/hrt.2008.143123, indexed in Pubmed: 18519550.

27. Kanjwal K, Kanjwal Y, Karabin B, et al. Clinical symptoms associated with asystolic or bradycardic responses on implantable loop recorder monitoring in patients with recurrent syncope. Int J Med Sci. 2009; 6(2): 106-110, indexed in Pubmed: 19381350.

28. Sud S, Klein GJ, Skanes AC, et al. Predicting the cause of syncope from clinical history in patients undergoing prolonged monitoring. Heart Rhythm. 2009; 6(2): 238-243, doi: 10.1016/j. hrthm.2008.10.035, indexed in Pubmed: 19187918.

29. Davis S, Westby M, Pitcher D, et al. Implantable loop recorders are cost-effective when used to investigate transient loss of consciousness which is either suspected to be arrhythmic or remains unexplained. Europace. 2012; 14(3): 402-409, doi: 10.1093/europace/eur343, indexed in Pubmed: 22071383.

30. Carpenter A, Frontera A. Smart-watches: a potential challenger to the implantable loop recorder? Europace. 2016; 18(6): 791-793, doi: 10.1093/europace/euv427, indexed in Pubmed: 26847074.

31. Edvardsson N, Wolff C, Tsintzos S, et al. Costs of unstructured investigation of unexplained syncope: insights from a micro-costing analysis of the observational PICTURE registry. Europace. 2015; 17(7): 1141-1148, doi: 10.1093/europace/euu412, indexed in Pubmed: 25759408. 